Check for updates

Cite this: RSC Adv., 2018, 8, 20434

Received 28th November 2017 Accepted 5th May 2018

DOI: $10.1039 / c 7 r a 12860 f$

rsc.li/rsc-advances

\section{Charged porous organic frameworks bearing heteroatoms with enhanced isosteric enthalpies of gas adsorption $\dagger$}

\author{
Guolong Xing, Tingting Yan, Saikat Das, Ling Ye and Kaiqi Ye (DD * \\ Charged porous organic frameworks containing heteroatoms were synthesized via a Yamamoto-type \\ Ullmann coupling reaction using potassium tetrakis(4-bromopyrazolyl)borate and tetrakis(4- \\ chlorophenyl)phosphonium bromide as monomers. For the first time, a monomer containing boron \\ atoms was successfully homocoupled to obtain a 3D charged porous organic framework. The \\ heteroatoms and charges in the porous organic frameworks help to increase the interaction between \\ the frameworks and the gases. Therefore the charged porous organic frameworks bearing heteroatoms \\ synthesized in the present study exhibit high isosteric enthalpies of gas adsorption which surpass those \\ of many other porous organic materials.
}

\section{Introduction}

Porous organic frameworks have lately attracted interest from scientists for applications such as gas separation, gas storage, catalysis and others because they have outstanding surface areas, high microporosities and remarkable physicochemical stabilities. ${ }^{1-6}$ Porous organic frameworks can be categorized into crystalline and amorphous frameworks. Crystalline porous organic frameworks such as covalent organic frameworks ${ }^{7-9}$ (COFs) retain structural order and well-defined porosities and incorporate building units linked by covalent bonds. Porous aromatic frameworks (PAFs) are porous networks with remarkably high surface areas and superb stabilities. ${ }^{\mathbf{1 0 - 1 3}}$ Porous organic frameworks used for gas adsorption/storage and selective gas separation are routinely characterized by their isosteric enthalpies of gas adsorption since they provide an understanding of the affinity of the frameworks for gases. The rapid increase of carbon dioxide in the environment due to industrialization has necessitated its adsorption, while hydrogen and methane are stored due to their use as clean fuels. Porous organic frameworks are being extensively used for the adsorption and storage of the aforementioned gases so it is important to know their isosteric enthalpies of gas adsorption.

As we know, a nitrogen atom in the framework is beneficial for $\mathrm{CO}_{2}$ adsorption and charges can make it easier for gas polarization to take place, therefore enhancing the interactions between the frameworks and gases. ${ }^{\mathbf{1 4 - 1 6}}$ From considering this, integrating charges and heteroatoms into porous organic

State Key Laboratory of Supramolecular Structure and Materials, College of Chemistry, Jilin University, Changchun 130012, P. R. China. E-mail: yekq@jlu.edu.cn $\uparrow$ Electronic supplementary information (ESI) available. See DOI: $10.1039 / \mathrm{c} 7 \mathrm{ra12860f}$ frameworks to synthesize charged porous organic frameworks bearing heteroatoms may help to increase the isosteric enthalpies of gas adsorption. Herein we introduce a series of charged porous organic frameworks, namely, B-POF, P-POF and BP-POF, bearing heteroatoms and synthesized via a Yamamototype Ullmann cross-coupling reaction from potassium tetrakis(4-bromopyrazolyl)borate and tetrakis(4-chlorophenyl) phosphonium bromide as monomers in different molar ratios. The heteroatoms and charges in these porous organic frameworks increase their isosteric enthalpies of gas adsorption. Thus, the charged porous organic frameworks show outstanding isosteric enthalpies for gas adsorption and outperform many other typical porous organic materials, such as PAF-1, COF-102, HCP-1 etc.

\section{Experimental}

\section{Materials and methods}

All of the starting materials were purchased from Aldrich and J\&K. $N, N$-Dimethylformamide (DMF) and 1,5-cyclooctadiene (COD) were dried over $\mathrm{CaH}_{2}$ and distilled under reduced pressure prior to their use. The FT-IR experiments with the charged porous organic frameworks began with the mixing of the charged porous organic frameworks and $\mathrm{KBr}$ which was followed by the forming of pellets from the mixture upon the application of pressure. Then a Shimadzu IRAffinity-1 FT-IR spectrophotometer was used to obtain the FT-IR spectra. The FT-IR measurements under vacuum were performed in a Shimadzu IRTracer-100 Fourier transform infrared spectrophotometer with a vacuum system by pressing the mixture of the charged porous organic frameworks and $\mathrm{KBr}$ into the porous alumina crucibles. To carry out TGA of the samples, the samples were loaded in an alumina pan and inserted inside a Shimadzu 
DTG-60 thermal analyzer which was heated to $900{ }^{\circ} \mathrm{C}$ at a heating rate of $10^{\circ} \mathrm{C} \mathrm{min}^{-1}$ in a dry air atmosphere with an air flow

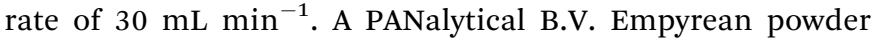
diffractometer using $\mathrm{Cu}-\mathrm{K} \alpha$ radiation, $40 \mathrm{kV}, 40 \mathrm{~mA}$ was employed to perform PXRD with the samples over the range $2 \theta=4.0-40.0^{\circ}$. The SEM images were taken with a JEOL JSM 6700 scanning electron microscope on silicon wafer. The TEM images were obtained with a JEOL JEM-2100F transmission electron microscope. $\mathrm{N}_{2}$ sorption isotherm experiments were carried out at $77 \mathrm{~K}$ using a MicroMeritics Tristar II 3020 surface area and pore size analyzer. The samples were degassed at $150{ }^{\circ} \mathrm{C}$ overnight under ultra-high vacuum prior to the $\mathrm{N}_{2}$ (99.999\% purity) sorption measurements. $\mathrm{H}_{2}(99.999 \%$ purity), $\mathrm{CO}_{2}$ (99.999\% purity) and $\mathrm{CH}_{4}$ (99.99\% purity) sorption isotherm measurements were carried out using the same analyzer that was used for $\mathrm{N}_{2}$ sorption isotherm measurements. To measure the free space, He of $99.999 \%$ purity was used and it was presumed that He was not adsorbed at any temperature in which the experiments were carried out. $\mathrm{H}_{2}$ isotherm measurements at $77 \mathrm{~K}$ were carried out in a liquid nitrogen bath, $\mathrm{H}_{2}$ isotherm measurements at $87 \mathrm{~K}$ were carried out in a liquid argon bath and $\mathrm{H}_{2}$ isotherms measurements at $195 \mathrm{~K}$ were carried out in an acetone/dry ice bath. $\mathrm{CO}_{2}$ and $\mathrm{CH}_{4}$ isotherm measurements at $273 \mathrm{~K}$ and 285 $\mathrm{K}$ were carried out in an ice-water bath and a 1,4-dioxane/dry ice bath, respectively.

\section{Synthetic procedures}

Synthesis of potassium tetrakis(4-bromopyrazolyl)borate. Potassium tetrakis(4-bromopyrazolyl)borate was synthesized in accordance with previous reports. ${ }^{17}$ Under an argon atmosphere, 4-bromopyrazole $(4.4091 \mathrm{~g}, 30 \mathrm{mmol})$ and potassium borohydride $(0.3236 \mathrm{~g}, 6 \mathrm{mmol})$ were mixed. Next, the mixture was heated gradually to $220{ }^{\circ} \mathrm{C}$ and kept for $11 \mathrm{~h}$. Afterwards it was cooled down to room temperature, and the product dissolved in THF and filtered using a Nylon membrane, and the solvent was removed under reduced pressure. The solid was subjected to a vacuum at $140{ }^{\circ} \mathrm{C}$ for $4 \mathrm{~h}$. Next, the solid was dissolved in ethanol and filtered using a PVDF membrane, and the solvent was removed under reduced pressure. The resulting solid was washed with chloroform and hot $n$-hexane, and dried under vacuum at $80{ }^{\circ} \mathrm{C}$ to obtain a white powder. Yield: $39 \% ;{ }^{1} \mathrm{H}$ NMR (400 MHz, DMSO-d 6 , $\delta$ ): 7.28 (s, 4H), $7.53(\mathrm{~s}, 4 \mathrm{H}) ;{ }^{13} \mathrm{C}$ NMR (101 MHz, DMSO-d $\left.{ }_{6}, \delta\right): 140.12,133.99,133.96,133.92,133.89$, 90.83, 90.81, 90.79, 90.77.

Synthesis of tetrakis(4-chlorophenyl)phosphonium bromide. Tetrakis(4-chlorophenyl)phosphonium bromide was synthesized in accordance with previous reports. ${ }^{18}$ Under an argon atmosphere, 1-bromo-4-chlorobenzene (0.8378 g, 4.376 $\mathrm{mmol}), \mathrm{Pd}(\mathrm{OAc})_{2}(0.0491 \mathrm{~g}, 5 \mathrm{~mol} \%)$ and tris( $p$-chloropheny1) phosphine $(1.6000 \mathrm{~g}, 4.376 \mathrm{mmol})$ were mixed and $4.4 \mathrm{~mL}$ of dry oxylene was added to the mixture. Next, the mixture was heated to $160{ }^{\circ} \mathrm{C}$ and refluxed for $16 \mathrm{~h}$. The mixture was then cooled down to room temperature and $20 \mathrm{~mL}$ of diethyl ether was added to it. The resulting suspension was stirred at room temperature for $2 \mathrm{~min}$. The precipitate was subsequently filtered and washed with $80 \mathrm{~mL}$ of diethyl ether. The product was dissolved in acetone and filtered using a PVDF membrane, and the solution was added to a large amount of diethyl ether. The precipitate was finally filtered and subjected to a vacuum at $50{ }^{\circ} \mathrm{C}$ to obtain a light yellow powder. Yield: $54 \%$; ${ }^{1} \mathrm{H}$ NMR (400 MHz, DMSO-d ${ }_{6}, \delta$ ): $7.90(\mathrm{dd}, J=8.8$, $2.9 \mathrm{~Hz}, 8 \mathrm{H}), 7.78$ (dd, $J=12.7,8.8 \mathrm{~Hz}, 8 \mathrm{H}) ;{ }^{13} \mathrm{C} \mathrm{NMR}(101 \mathrm{MHz}$, DMSO-d $\left._{6}, \delta\right): 141.21,141.17,136.67,136.54,130.70,130.57$, 116.31, 115.39.

Synthesis of the charged porous organic frameworks bearing heteroatoms. Potassium tetrakis(4-bromopyrazolyl)borate and tetrakis(4-chlorophenyl)phosphonium bromide (1:0, $3: 1$ and $0: 1 \mathrm{~mol} / \mathrm{mol}$ ) were dissolved in $N, N$-dimethylformamide (DMF, $33 \mathrm{~mL}, 0.422 \mathrm{~mol})$ to prepare the monomer mixture. After this, 1,5-cyclooctadiene $(0.224 \mathrm{~mL}, 1.824 \mathrm{mmol})$ was added to a solution of bis(1,5-cyclooctadiene)nickel(0) (500.4 mg, 1.824 $\mathrm{mmol}$ ) and 2,2'-bipyridyl (284.9 $\mathrm{mg}, 1.824 \mathrm{mmol})$ in anhydrous DMF $(5 \mathrm{~mL})$. The mixture was subsequently heated at $80^{\circ} \mathrm{C}$ for $1 \mathrm{~h}$. Next the monomer mixture solution was added to the resulting solution at $80{ }^{\circ} \mathrm{C}$. The whole mixture was stirred at $80{ }^{\circ} \mathrm{C}$ for $3 \mathrm{~d}$. Then the mixture was cooled down to room temperature following which an aqueous solution of $\mathrm{HCl}(\mathrm{pH}=$ 3) was added to it. The solution was then stirred for $36 \mathrm{~h}$ (keep $\mathrm{pH}=3$ ), filtered and the residue washed with distilled water, ethanol and chloroform and dried under vacuum to obtain the charged porous organic frameworks, namely, B-POF, P-POF and BP-POF.

\section{Results and discussion}

\section{Synthesis}

The synthesis of the charged porous organic frameworks was carried out via a Yamamoto-type Ullmann coupling reaction using potassium tetrakis(4-bromopyrazolyl)borate and tetrakis(4-chlorophenyl)phosphonium bromide as monomers. Scheme 1 illustrates the synthesis technique adopted to obtain $\mathrm{B}-\mathrm{POF}, \mathrm{P}-\mathrm{POF}$ and BP-POF.

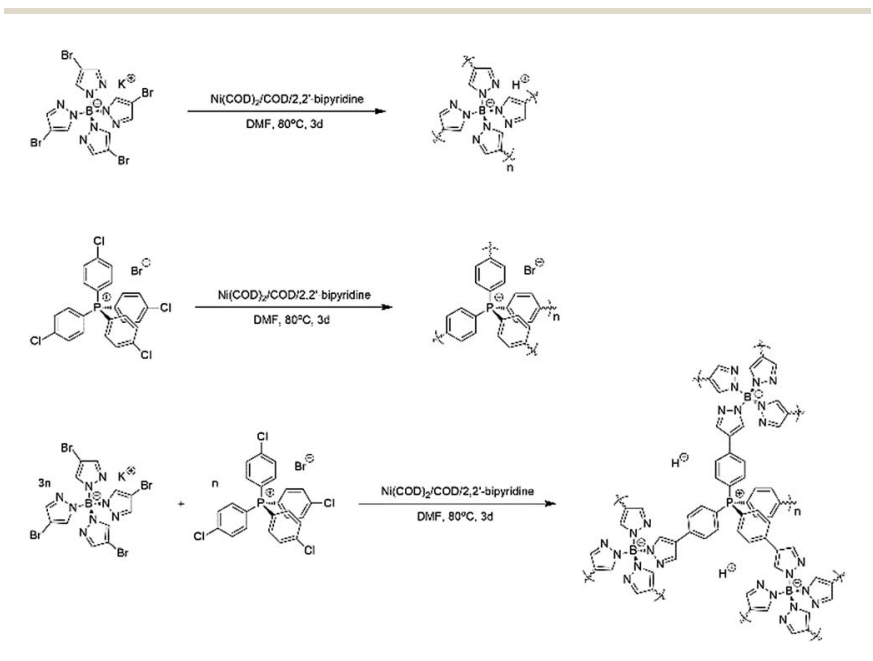

Scheme 1 Schematic representation of the synthesis of the porous organic frameworks, namely, (top) B-POF, (middle) P-POF, and (bottom) BP-POF. 


\section{Characterization}

The structures of the charged porous frameworks were confirmed by their Fourier transform infrared (FT-IR) spectra, as shown in Fig. 1. The bands at about 611 and $760 \mathrm{~cm}^{-1}$ corresponding to the stretching vibrations of the $\mathrm{C}-\mathrm{Br}$ and $\mathrm{C}-\mathrm{Cl}$ bonds, respectively, decreased in the FTIR spectra of the porous organic frameworks, as compared to the FTIR spectra of the monomers. This substantiates the fact that the coupling reaction had occurred. Compared with the FT-IR spectra measured under vacuum (Fig S1-S3†), with the vacuum time increasing, the stretching bands appearing around $\sim 3406 \mathrm{~cm}^{-1}$ are less intense, which can be attributed to the presence of water molecules. The bands which appear around $\sim 3030 \mathrm{~cm}^{-1}$ in the FT-IR spectra of BP-POF, P-POF and the monomer tetrakis(4chlorophenyl)phosphonium bromide can be assigned to the vibration of the $\mathrm{C}-\mathrm{H}$ bond in the benzene ring, while the bands at about $\sim 3134 \mathrm{~cm}^{-1}$ and $\sim 3423 \mathrm{~cm}^{-1}$ which appear in the spectra of B-POF, BP-POF and the monomer potassium tetrakis(4-bromopyrazolyl)borate correspond to the $\mathrm{C}-\mathrm{H}$ bond and the $\mathrm{N}-\mathrm{H}$ bond in the pyrazole ring.

To further investigate the structure, the charged porous organic frameworks were characterized using solid-state ${ }^{11} \mathrm{~B}$ and ${ }^{31} \mathrm{P}$ NMR. As shown in Fig $\mathrm{S} 4-\mathrm{S} 5, \dagger$ the ${ }^{31} \mathrm{P}$ NMR spectrum of BP-POF shows two signals at $23.2 \mathrm{ppm}$ and $8.5 \mathrm{ppm}$ while that of P-POF shows two signals at $22.8 \mathrm{ppm}$ and $9.1 \mathrm{ppm}$, which can
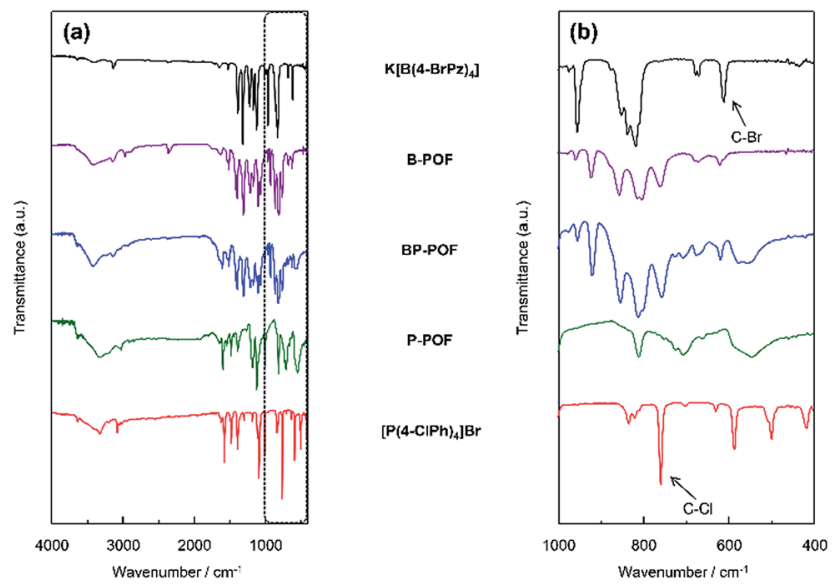

Fig. 1 (a) FTIR spectra of $\mathrm{K}\left[\mathrm{B}(4-\mathrm{BrPz})_{4}\right], \mathrm{B}-\mathrm{POF}, \mathrm{BP}-\mathrm{POF}, \mathrm{P}-\mathrm{POF}$ and $\left[\mathrm{P}(4-\mathrm{ClPh})_{4}\right] \mathrm{Br}$; (b) FT-IR spectra in detail from 400 to $1000 \mathrm{~cm}^{-1}$. be attributed to the quaternary phosphonium and tertiary phosphine atoms, respectively. The tertiary phosphine atoms can be generated by the cleaving of the $\mathrm{P}-\mathrm{C}$ bond during polymerization. ${ }^{18}$ However, unfortunately ${ }^{11} \mathrm{~B}$ NMR spectra can't be obtained owing to the low content of boron in the frameworks.

The scanning electron microscopy (SEM) images of the porous organic frameworks are shown in Fig S6. $\dagger$ They show that the porous frameworks do not have a well-defined shape. The transmission electron microscopy (TEM) images of the porous organic frameworks are shown in Fig. 2. They reveal the amorphous nature of the porous frameworks.

In order to explore the short-range and long-range orders of the samples, powder X-ray diffraction (PXRD) patterns of the samples were obtained. As shown in Fig. S7, $\dagger$ the PXRD patterns of the porous frameworks corroborate the amorphous nature (and thereby the lack of long-range order) of the materials. This is in accordance with the results from TEM. However, B-POF, for which a broad diffraction peak at $10.7^{\circ}$ was revealed, likely has some short-range order.

The thermal and chemical stabilities of the porous organic frameworks were also investigated. Fig S $\$$ † shows the thermogravimetric analysis (TGA) plots of the samples. Of the materials, P-POF showed the highest stability with a 5\% weight loss at $460{ }^{\circ} \mathrm{C}$ followed by B-POF and BP-POF with weight losses of $5 \%$ at $312{ }^{\circ} \mathrm{C}$ and $269^{\circ} \mathrm{C}$ in an air atmosphere, respectively. Furthermore, the samples also exhibited high chemical stabilities, and were insoluble in water and common organic solvents such as hexanes, chloroform, ethanol and acetone.

\section{Gas adsorption}

The specific surface areas and porosities of the samples were explored using nitrogen sorption measurements at $77 \mathrm{~K}$. As shown in Fig. 3a, in the low pressure region $\left(p / p_{0}<0.1\right)$, the isotherms show steep adsorption which confirms the presence of micropores. The three isotherms show a hysteresis loop, which means that mesopores are present in the frameworks. However, it can also be observed that the hysteresis loop extends to a low relative pressure. This may be attributed to swelling in the porous organic framework. ${ }^{18}$ The BrunauerEmmett-Teller (BET) surface areas of the porous frameworks were calculated from the sorption isotherms to be $515 \mathrm{~m}^{2} \mathrm{~g}^{-1}$, $629 \mathrm{~m}^{2} \mathrm{~g}^{-1}$ and $223 \mathrm{~m}^{2} \mathrm{~g}^{-1}$ for B-POF, P-POF, and BP-POF,
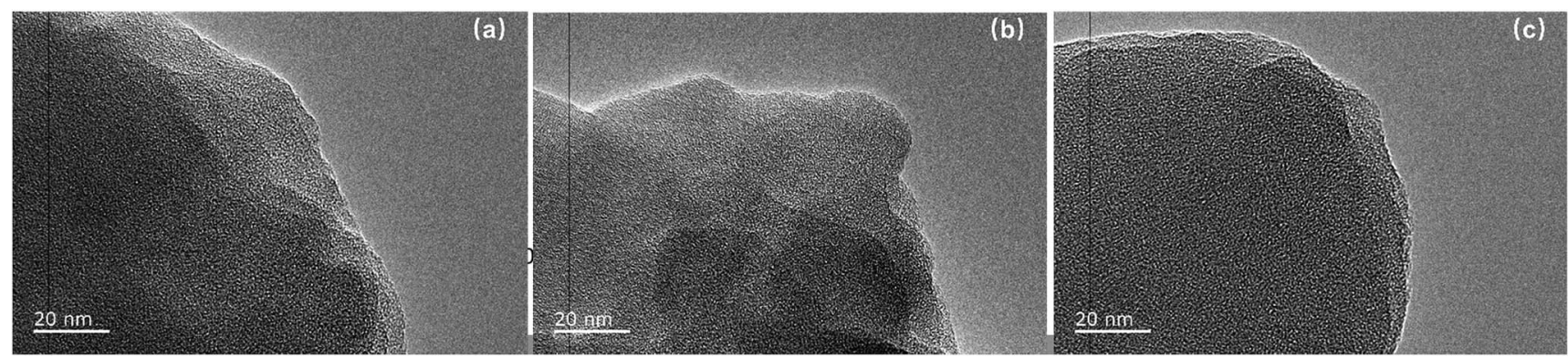

Fig. 2 TEM images of (a) B-POF, (b) BP-POF and (c) P-POF. 

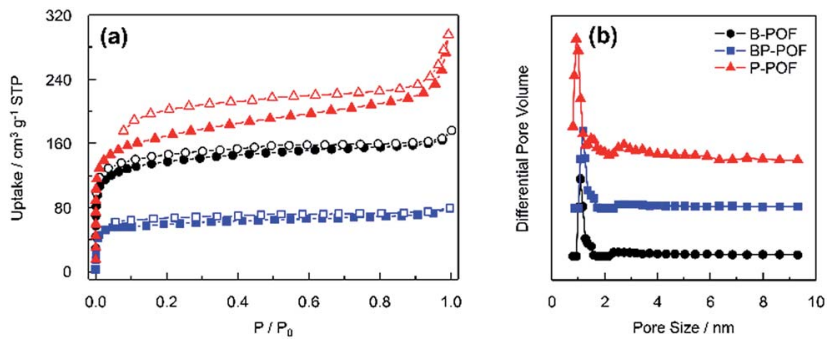

Fig. 3 (a) $N_{2}$ sorption isotherms and (b) corresponding pore size distribution of B-POF (circles), BP-POF (squares), and P-POF (triangles) at $77 \mathrm{~K}$ (solid symbols, adsorption; open symbols, desorption).

respectively (Table 1). Fig. $3 \mathrm{~b}$ shows the pore size distribution of the samples calculated by the density functional theory (DFT) method. It shows dominant pore diameters centered at about $1.18 \mathrm{~nm}, 1.18 \mathrm{~nm}$ and $0.93 \mathrm{~nm}$ corresponding to B-POF, BP$\mathrm{POF}$, and P-POF, respectively. The total pore volumes for $\mathrm{B}-$ POF, BP-POF, and P-POF are $0.13 \mathrm{~cm}^{3} \mathrm{~g}^{-1}, 0.08 \mathrm{~cm}^{3} \mathrm{~g}^{-1}$ and $0.18 \mathrm{~cm}^{3} \mathrm{~g}^{-1}$. BP-POF shows a low surface area value and a low pore volume which may be attributed to a low level of polymerization caused by the different reactivity of $\mathrm{Br}$ and $\mathrm{Cl}$.

$\mathrm{H}_{2}, \mathrm{CO}_{2}$ and $\mathrm{CH}_{4}$ are apolar molecules with kinetic diameters $2.90,3.30$, and $3.80 \AA$, respectively. In addition, the order of polarizability is $\mathrm{CO}_{2}>\mathrm{CH}_{4}>\mathrm{H}_{2}$, which means that $\mathrm{CO}_{2}$ is easier to polarize than the other two. In order to investigate the affinities of the samples, adsorption isotherms were collected, and isosteric enthalpies of gas adsorption $\left(Q_{\mathrm{st}}\right)$ were calculated using the Clausius-Clapeyron equation from the adsorption isotherms at different temperatures.

The $\mathrm{H}_{2}$ isotherms at $77 \mathrm{~K}$ and 1 bar exhibit maximum uptakes of $103.3 \mathrm{~cm}^{3} \mathrm{~g}^{-1}, 102.8 \mathrm{~cm}^{3} \mathrm{~g}^{-1}$, and $100.0 \mathrm{~cm}^{3} \mathrm{~g}^{-1}$ for B-POF, P-POF, and BP-POF, respectively (Fig. 4a). The $Q_{\text {st }}$ values for $\mathrm{H}_{2}$ adsorption for B-POF, P-POF, and BP-POF were found to be $8.5,7.8$, and $7.9 \mathrm{~kJ} \mathrm{~mol}^{-1}$ (Fig. 4b). These values are higher than those for numerous porous polymers. PAF-1 with a high BET surface area of up to $5600 \mathrm{~m}^{2} \mathrm{~g}^{-1}$ exhibited a $Q_{\mathrm{st}}$ value of $4.6 \mathrm{~kJ} \mathrm{~mol}^{-1}$ for $\mathrm{H}_{2} \cdot{ }^{12} \mathrm{COF}-102$, with a BET surface area as high as $3620 \mathrm{~m}^{2} \mathrm{~g}^{-1}$, shows a $Q_{\text {st }}$ value of $3.9 \mathrm{~kJ} \mathrm{~mol}^{-1}$ for $\mathrm{H}_{2} \cdot{ }^{19} \mathrm{JUC}$ Z7-10, which also has a high BET surface area, exhibited a $Q_{\mathrm{st}}$ value in the range $5.6-6.9 \mathrm{~kJ} \mathrm{~mol}^{-1} .^{20} \mathrm{GNF}-1$ with a similar BET surface area $\left(679 \mathrm{~m}^{2} \mathrm{~g}^{-1}\right)$ gave a $Q_{\text {st }}$ value of $7.7 \mathrm{~kJ} \mathrm{~mol}^{-1} .^{21}$ Although $\mathrm{H}_{2}$ is not easy to polarize, the charged boron atoms and phosphorus atoms in the frameworks still enhance the
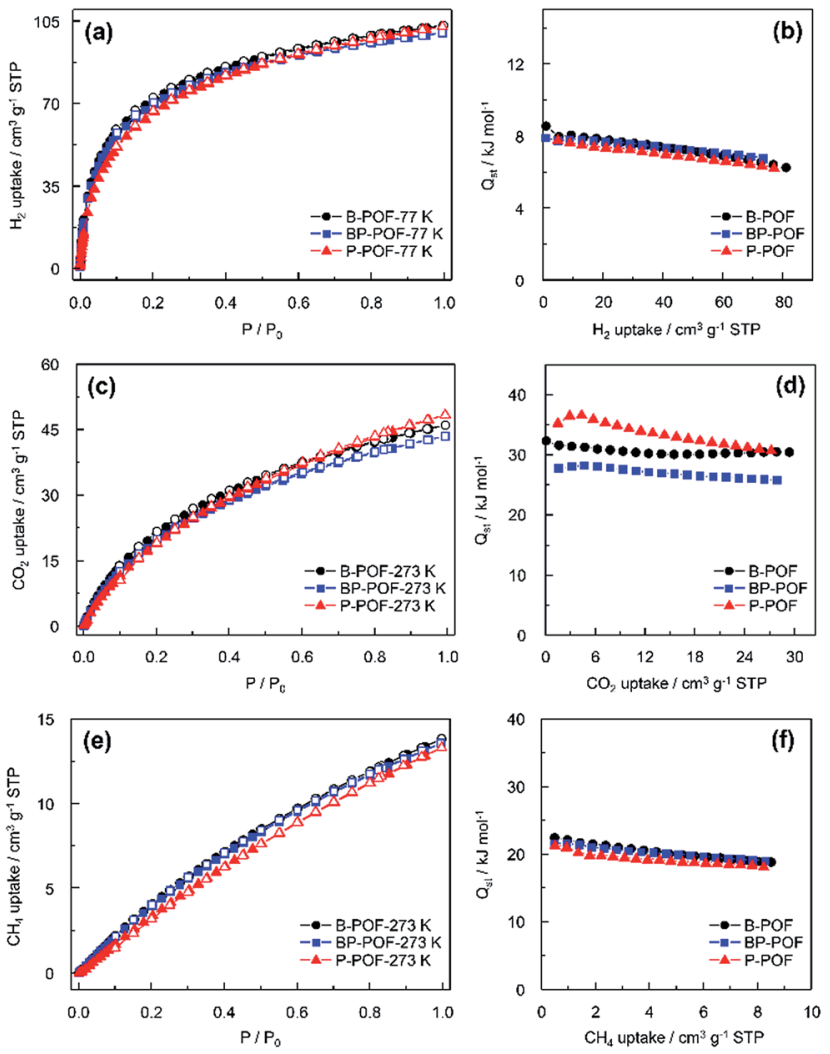

Fig. 4 (a) $\mathrm{H}_{2}$ sorption isotherms and (b) $Q_{\mathrm{stH}_{2}}$ of B-POF (circles), BPPOF (squares), and P-POF (triangles) at $77 \mathrm{~K}$; (c) $\mathrm{CO}_{2}$ sorption isotherms and (d) $Q_{\mathrm{stCO}_{2}}$ of B-POF (circles), BP-POF (squares), and PPOF (triangles) at $273 \mathrm{~K}$; (e) $\mathrm{CH}_{4}$ sorption isotherms and (f) $Q_{\mathrm{stCH}}$ of $\mathrm{B}$ POF (circles), BP-POF (squares), and P-POF (triangles) at $273 \mathrm{~K}$ (solid symbols, adsorption; open symbols, desorption).

polarization effects of the frameworks for gases, and therefore help to increase the interactions between the frameworks and $\mathrm{H}_{2}$, which thus exhibit high isosteric enthalpies.

The $\mathrm{CO}_{2}$ isotherms of B-POF, P-POF, and BP-POF are shown in Fig. 4c. At $273 \mathrm{~K}$, the $\mathrm{CO}_{2}$ uptake capacities of the porous organic frameworks are $46.0 \mathrm{~cm}^{3} \mathrm{~g}^{-1}, 48.4 \mathrm{~cm}^{3} \mathrm{~g}^{-1}$ and $43.5 \mathrm{~cm}^{3}$ $\mathrm{g}^{-1}$ at 1 bar. The $Q_{\mathrm{st}}$ values for $\mathrm{CO}_{2}$ were calculated from the adsorption data collected at $273 \mathrm{~K}, 285 \mathrm{~K}$ and $295 \mathrm{~K}$. The three POFs exhibit high $Q_{\text {st }}$ values, especially B-POF and P-POF. These values are comparable to, if not greater than, the values reported for most porous polymers including HCP$1\left(\sim 23.5 \quad \mathrm{~kJ} \quad \mathrm{~mol}^{-1}\right),{ }^{22} \quad \mathrm{CTF}-1\left(\sim 27.5 \quad \mathrm{~kJ} \quad \mathrm{~mol}^{-1}\right),{ }^{23} \quad$ BILP-

Table 1 Summary of the structural properties and the isosteric enthalpies of $\mathrm{H}_{2}, \mathrm{CO}_{2}$ and $\mathrm{CH}_{4}$ adsorption for the synthesized charged porous organic frameworks

\begin{tabular}{|c|c|c|c|c|c|c|c|c|}
\hline POFs & $\begin{array}{l}\mathrm{S}_{\mathrm{BET}}{ }^{a} \\
\left(\mathrm{~m}^{2} \mathrm{~g}^{-1}\right)\end{array}$ & $\begin{array}{l}\text { Pore size } \\
(\mathrm{nm})\end{array}$ & $\begin{array}{l}V_{\text {micro }} \\
\left(\mathrm{cm}^{3} \mathrm{~g}^{-1}\right)\end{array}$ & $\begin{array}{l}V_{\text {meso }} \\
\left(\mathrm{cm}^{3} \mathrm{~g}^{-1}\right)\end{array}$ & $\begin{array}{l}V_{\text {total }} \\
\left(\mathrm{cm}^{3} \mathrm{~g}^{-1}\right)\end{array}$ & $\begin{array}{l}Q_{\mathrm{stH}_{2}} \\
\left(\mathrm{~kJ} \mathrm{~mol}^{-1}\right)\end{array}$ & $\begin{array}{l}Q_{\mathrm{stCO}_{2}} \\
\left(\mathrm{~kJ} \mathrm{~mol}^{-1}\right)\end{array}$ & $\begin{array}{l}Q_{\mathrm{stCH}_{4}} \\
\left(\mathrm{~kJ} \mathrm{~mol}^{-1}\right)\end{array}$ \\
\hline B-POF & 515 & 1.18 & 0.13 & 0.03 & 0.16 & 8.5 & 32.3 & 22.4 \\
\hline P-POF & 223 & $0.93,1.48$ and 2.73 & 0.18 & 0.08 & 0.26 & 7.8 & 35.2 & 21.4 \\
\hline
\end{tabular}

${ }^{a}$ The BET surface areas were determined in the partial pressure $\left(p / p_{0}\right)$ range $0.01-0.1$. 
$1\left(26.5 \mathrm{~kJ} \mathrm{~mol}^{-1}\right),{ }^{24}$ PMOP-1(18.5 kJ mol $\left.{ }^{-1}\right),{ }^{25}$ PCTF $1-7(25-$ $\left.30 \mathrm{~kJ} \mathrm{~mol}^{-1}\right),{ }^{26} \mathrm{~N}_{4} \mathrm{CMP} 1-4\left(25.5-35.1 \mathrm{~kJ} \mathrm{~mol}^{-1}\right),{ }^{27}$ polycarbazole CPOP-1(24.5-30.2 kJ mol $\left.{ }^{-1}\right)^{28}$ and P-THIDT $\left(29 \mathrm{~kJ} \mathrm{~mol}^{-1}\right) .{ }^{29}$ The high value for $Q_{\mathrm{st}}$ for $\mathrm{CO}_{2}$ adsorption could perhaps be attributed to the presence of charges and nitrogen atoms in the POF. The coexistence of positive and negative charges in BP-POF are not beneficial for polarizing $\mathrm{CO}_{2}$ molecules, therefore BP-POF exhibits a relatively lower isosteric enthalpy than B-POF and P-POF.

As a promising energy source in the future, methane is highly popular due to its high ratio of hydrogen to carbon. Therefore, the $\mathrm{CH}_{4}$ sorption behavior of B-POF, P-POF, and BP-POF were also investigated. As shown in Fig. 4 e, the $\mathrm{CH}_{4}$ isotherms are reversible and show maximum uptakes of 13.8 $\mathrm{cm}^{3} \mathrm{~g}^{-1}, 13.3 \mathrm{~cm}^{3} \mathrm{~g}^{-1}$, and $13.6 \mathrm{~cm}^{3} \mathrm{~g}^{-1}$, respectively. The $Q_{\mathrm{st}}$ of $\mathrm{CH}_{4}$ for B-POF, P-POF, and BP-POF were also calculated and the values are $22.4,21.4$ and $21.5 \mathrm{~kJ} \mathrm{~mol}^{-1}$. These $Q_{\mathrm{st}}$ values are similar and surpass those of PAF-1 $\left(14.0 \mathrm{~kJ} \mathrm{~mol}^{-1}\right){ }^{12}$ PMOP-1 $\left(\sim 11 \mathrm{~kJ} \mathrm{~mol}^{-1}\right),{ }^{25}$ JUC-Z7-Z10(15.7-16.0 kJ mol $\left.{ }^{-1}\right)^{20}$ and BILP-3 $\left(16.6 \mathrm{~kJ} \mathrm{~mol}^{-1}\right),{ }^{30}$ and are similar to pre$\operatorname{GNF}\left(22.7 \mathrm{~kJ} \mathrm{~mol}^{-1}\right),{ }^{21}$ P-DTBDT $\left(25 \mathrm{~kJ} \mathrm{~mol}^{-1}\right)^{29}$ and CTF$\operatorname{DCBT}\left(22.8 \mathrm{~kJ} \mathrm{~mol}^{-1}\right){ }^{31}$ These high $Q_{\text {st }}$ values can also be attributed to the charged boron atoms or phosphorus atoms existing in the frameworks which favor $\mathrm{CH}_{4}$ polarization. This therefore increases the interactions between the frameworks and $\mathrm{CH}_{4}$ molecules.

Isosteric enthalpies of gas adsorption $\left(Q_{\mathrm{st}}\right)$ are useful values that characterize porous organic frameworks in their affinities for gases such as $\mathrm{CO}_{2}, \mathrm{H}_{2}, \mathrm{CH}_{4}$ etc. The porous organic frameworks synthesized in the present study possibly owe their outstanding isosteric enthalpies of gas adsorption to the heteroatoms and charges in the porous organic frameworks. The nitrogen atoms in the frameworks favor $\mathrm{CO}_{2}$ adsorption, and charges in the frameworks make the gas molecules much easier to polarize, which helps to increase the interactions between the frameworks and the gases. Thus, the POFs may have another potential application in adsorbing other gas molecules which are easier to polarize.

\section{Conclusions}

To summarize, porous organic frameworks which comprised heteroatoms were synthesized by a Yamamoto-type Ullmann coupling reaction using potassium tetrakis(4-bromopyrazolyl) borate and tetrakis(4-chlorophenyl)phosphonium bromide as monomers. These porous organic frameworks were found to exhibit high isosteric enthalpies of gas adsorption which may be attributed to the presence of heteroatoms and charges in the porous frameworks. Therefore, the charged porous organic frameworks bearing heteroatoms may effectively store the gases in ambient environments owing to their high affinities for the gases and release the gases when needed due to the reversible physical adsorption process. This study may motivate an exploration of the potential for more charged porous organic frameworks bearing heteroatoms for applications in gas sorption/storage and separation.

\section{Conflicts of interest}

There are no conflicts to declare.

\section{Acknowledgements}

This work was supported by the National Key R\&D Program of China (2016YFB0401001).

\section{Notes and references}

1 X. Feng, X. Ding and D. Jiang, Chem. Soc. Rev., 2012, 41, 6010-6022.

2 P. J. Waller, F. Gándara and O. M. Yaghi, Acc. Chem. Res., 2015, 48, 3053-3063.

3 Y. Xu, S. Jin, H. Xu, A. Nagai and D. Jiang, Chem. Soc. Rev., 2013, 42, 8012-8031.

4 S. Ren, M. J. Bojdys, R. Dawson, A. Laybourn, Y. Z. Khimyak, D. J. Adams and A. I. Cooper, Adv. Mater., 2012, 24, 23572361.

5 T. Ben and S. Qiu, CrystEngComm, 2013, 15, 17-26.

6 S. Das, P. Heasman, T. Ben and S. Qiu, Chem. Rev., 2017, 117, 1515-1563.

7 A. P. Côté, A. I. Benin, N. W. Ockwig, M. O'Keeffe, A. J. Matzger and O. M. Yaghi, Science, 2005, 310, 1166-1170.

8 H. M. El-Kaderi, J. R. Hunt, J. L. Mendoza-Cortés, A. P. Côté, R. E. Taylor, M. O'Keeffe and O. M. Yaghi, Science, 2007, 316, 268-272.

9 F. J. Uribe-Romo, C. J. Doonan, H. Furukawa, K. Oisaki and O. M. Yaghi, J. Am. Chem. Soc., 2011, 133, 11478-11481.

10 T. Ben, H. Ren, S. Ma, D. Cao, J. Lan, X. Jing, W. Wang, J. Xu, F. Deng, J. M. Simmons, S. Qiu and G. Zhu, Angew. Chem., Int. Ed., 2009, 48, 9457-9460.

11 T. Ben, C. Pei, D. Zhang, J. Xu, F. Deng, X. Jing and S. Qiu, Energy Environ. Sci., 2011, 4, 3991-3999.

12 T. Ben, Y. Li, L. Zhu, D. Zhang, D. Cao, Z. Xiang, X. Yao and S. Qiu, Energy Environ. Sci., 2012, 5, 8370-8376.

13 C. Pei, T. Ben and S. Qiu, Mater. Horiz., 2015, 2, 11-21.

14 S. Fischer, A. Schimanowitz, R. Dawson, I. Senkovska, S. Kaskel and A. Thomas, J. Mater. Chem. A, 2014, 2, 11825-11829.

15 O. Buyukcakir, S. H. Je, S. N. Talapaneni, D. Kim and A. Coskun, ACS Appl. Mater. Interfaces, 2017, 9, 7209-7216.

16 (a) M. Zhang, Z. Perry, J. Park and H.-C. Zhou, Polymer, 2014, 55, 335-339; (b) W. Lu, J. P. Sculley, D. Yuan, R. Krishna and H.-C. Zhou, J. Phys. Chem. C, 2013, 117, 4057-4061.

17 M. Onishi, Y. Arikawa, M. Yamaguchi, T. Nagano, T. Inoue, A. Terasoba, S. Matsuo, M. Nakagawa, H. Kawano, J. Nagaoka, K. Umakoshi and M. Furukawa, J. Organomet. Chem., 2012, 700, 135-141.

18 Q. Zhang, S. Zhang and S. Li, Macromolecules, 2012, 45, 2981-2988.

19 H. Furukawa and O. M. Yaghi, J. Am. Chem. Soc., 2009, 131, 8875-8883.

20 C. Pei, T. Ben, Y. Li and S. Qiu, Chem. Commun., 2014, 50, 6134-6136.

21 Y. Byun and A. Coskun, Chem. Mater., 2015, 27, 2576-2583. 
22 C. F. Martin, E. Stöckel, R. Clowes, D. J. Adams, A. I. Cooper, J. J. Pis, F. Rubiera and C. Pevida, J. Mater. Chem., 2011, 21, 5475-5483.

23 Y. Zhao, K. X. Yao, B. Teng, T. Zhang and Y. A. Han, Energy Environ. Sci., 2013, 6, 3684-3692.

24 M. G. Rabbani and H. M. El-Kaderi, Chem. Mater., 2011, 23, 1650-1653.

25 J. Liu, Y. Liu, X. Jiang, Y. Luo and Y. Lyu, Microporous Mesoporous Mater., 2017, 250, 203-209.

26 A. Bhunia, I. Boldog, A. Möller and C. Janiak, J. Mater. Chem. A, 2013, 1, 14990.
27 G. Li, L. Qin, C. Yao and Y. Xu, Sci. Rep., 2017, 7, 15394.

28 Q. Chen, M. Luo, P. Hammershoj, D. Zhou, Y. Han, B. W. Laursen, C. G. Yan and B. H. Han, J. Am. Chem. Soc., 2012, 134, 6084-6087.

29 H. Liu, Q. Li, Q. Li, W. Jin, X. Li, A. Hameed and S. Qiao, Poly. Chem., 2017, 8, 6733-6740.

30 M. G. Rabbani, T. E. Reich, R. M. Kassab, K. T. Jackson and H. M. El-Kaderi, Chem. Commun., 2012, 48, 1141-1143.

31 K. Wang, Y. Tang, Q. Jiang, Y. Lan, H. Huang, D. Liu and C. Zhong, J. Energy Chem., 2017, 26, 902-908. 